\title{
Status of perturbative QCD calculations for deep-inelastic scattering and related processes
}

\author{
Sven-Olaf Moch \\ Deutsches Elektronen-Synchrotron DESY \\ Platanenallee 6, D-15738 Zeuthen, Germany
}

\begin{abstract}
We give a brief overview of the status of perturbative QCD calculations for deep-inelastic scattering. The radiative corrections to the Wilson coefficients are generally available to next-to-next-to-leading order in QCD and we address the accuracy of the strong coupling constant, the parton distributions of the nucleon and the heavy quark masses which is required for precision predictions. We also discuss related processes at hadron colliders such as Higgs production via weak boson fusion which can be described through structure functions of deep-inelastic scattering, building upon an approximate, although very accurate, factorization of the perturbative QCD corrections.
\end{abstract}

Keywords:

\section{Deep-inelastic scattering}

Deep-inelastic scattering (DIS) and the observed scaling violations have been central to the formulation of Quantum Chromodynamics (QCD) as the gauge theory of the strong interactions $[1,2]$. Over the decades the available high precision experimental data from lepton- and neutrino scattering off fixed targets at CERN, FNAL and SLAC as well as from electronproton collisions at the HERA collider at DESY have successfully probed QCD in a wide kinematical range. The key observables are inclusive structure functions or differential cross sections which provide the theoretical description of the hard hadronic interactions in the QCD improved parton model. Precision predictions in perturbative QCD rest on the fact that we can separate the sensitivity to dynamics from different scales, i.e., the physics at scale of the nucleon mass from hard highenergy scattering at a large scale $Q^{2}$. In Fig. 1 this is depicted for lepton-proton DIS in the one-boson exchange approximation, see e.g., Ref. [3] for the definitions of the kinematic variables.

The factorization at a scale $\mu$ allows to express for instance the unpolarized inclusive structure functions

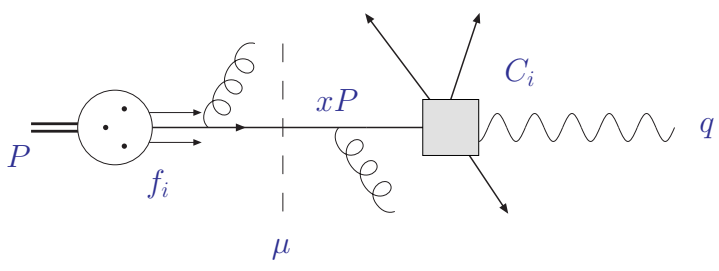

Figure 1: QCD factorization of the cross section for the scattering of a deeply virtual boson with (space-like) momentum $q\left(-q^{2}=Q^{2}>0\right)$ off a nucleon with momentum $P$ in their center-of-mass frame, see Eq. (1).

$F_{k}(k=2,3, L)$ as convolutions of parton distributions (PDFs) $f_{i}(i=q, \bar{q}, g)$ and short-distance Wilson coefficient functions $C_{k, i}$,

$$
\begin{aligned}
& F_{k}\left(x, Q^{2}\right)= \\
& \sum_{i=q, \bar{q}, g} \int_{x}^{1} d z f_{i}\left(\frac{x}{z}, \mu^{2}\right) C_{k, i}\left(z, Q^{2}, \alpha_{s}(\mu), \mu^{2}\right),
\end{aligned}
$$

up to corrections of higher twist $O\left(1 / Q^{2}\right)$. The coefficient functions $C_{k, i}$ are calculable perturbatively in QCD 
in powers of the strong coupling constant $\alpha_{s}$,

$$
\begin{aligned}
& C= \\
& \quad C^{(0)}+\alpha_{s} C^{(1)}+\alpha_{s}{ }^{2} C^{(2)}+\alpha_{s}{ }^{3} C^{(3)}+\ldots,
\end{aligned}
$$

with the expansion coefficients $C^{(0)}$ denoted as the leading order (LO), $C^{(1)}$ the next-to-leading order (NLO) and so on. The PDFs $f_{i}$ in Eq. (1) describe the fraction $x=Q^{2} /(2 P \cdot q)$ of the nucleon momentum carried by the quark or gluon, cf. Fig. 1. They are non-perturbative parameters like the strong coupling constant $\alpha_{s}\left(M_{Z}\right)$ and, if required, masses of heavy quarks, see the discussion in Sec. 2. QCD factorization has also been established for semi-inclusive deep-inelastic scattering as well as for DIS jet production, where the respective cross sections $d^{2} \sigma / d x d Q^{2}$ are subject to a decomposition analogous to Eq. (1). Although, in the latter cases, the process dependent hard parton scattering cross sections need to be augmented by additional prescriptions for the final state partons, e.g., a jet algorithm or fragmentation functions.

Over the years QCD predictions for DIS observables have reached an unprecedented level of precision. The Wilson coefficients in Eq. (2) have been computed to higher orders in perturbation theory so that the effect of radiative corrections on those observables is well understood and largely under control. In the case of inclusive unpolarized DIS, the coefficient functions $C_{k, i}$ are available to $O\left(\alpha_{s}{ }^{2}\right)$, i.e. to NNLO [4, 5, 6, 7]. For photon and charged current $W^{ \pm}$-boson exchange, even the hard corrections at order $O\left(\alpha_{s}{ }^{3}\right)$ have been obtained [8, 9]. For completeness, we also mention that the approach to polarized DIS is completely analogous. In particular for the structure function $g_{1}$ one may apply the obvious replacements $f_{i} \rightarrow \Delta f_{i}$ and $C_{k, i} \rightarrow \Delta C_{g_{1}, i}$ in Eqs. (1) and (2). At NNLO, the coefficient functions $\Delta C_{g_{1}, i}$ are available from [10].

In specific kinematical limits such as at high energies (small- $x$ ) or near threshold (large $x$ ) resummations of logarithmic corrections to all orders in perturbation theory exist to improve the perturbative predictions. Soft gluon resummation, i.e. in the limit $x \rightarrow 1$, has been addressed to next-to-next-to-next-to-leading logarithmic $\left(\mathrm{N}^{3} \mathrm{LL}\right)$ accuracy in $[11,12]$. The resummation at high energies based on the concept of $k_{t}$-factorization has been carried out to NLL [13].

Another important issue concerns the production of heavy quarks in DIS, which introduces a second hard scale, the heavy quark mass $m_{q}$. The pair-production of charm-quarks accounts for a considerable part of the inclusive DIS cross section measured at HERA, especially at small $x$, through the photon-gluon fusion process $\gamma^{*} g \rightarrow c \bar{c} X$, at not too large values of $Q^{2}$. The
Wilson coefficients of the heavy-quark structure functions are known exactly to NLO since long, both for the neutral [14] and the charged current [15] case, while the NNLO results are, at present, approximate only and based on the logarithmically enhanced terms near threshold $[16,17,18]$.

For semi-inclusive observables, the QCD corrections are typically known to NLO. This corresponds to $O\left(\alpha_{s}{ }^{n+1}\right)$ since the underlying Born cross section behaves as $d^{2} \sigma^{(0)} / d x d Q^{2} \sim O\left(\alpha_{s}{ }^{n}\right)$ due to the $n$ additional final state partons. Processes considered include the electro-production of hadrons with high transverse momentum $[19,20]$ or DIS $n$-jet cross sections. The latter is subject of continuing interest since the beginning of HERA operations, see e.g., [21, 22, 23, 24]. The calculation of the full NNLO QCD corrections for DIS 1 -jet inclusive production is still in progress (see [25] and references therein).

The available higher order QCD predictions for DIS generally display the anticipated features of apparent convergence of the perturbative expansion and stability under scale variation in a typical range, say $Q / 2 \leq$ $\mu \leq 2 Q$. The latter property is commonly used as a means to estimate the residual theory uncertainty, based on the fact that physical observables like the structure functions $F_{k}\left(x, Q^{2}\right)$ in Eq. (1) cannot depend on the factorization scale. In the perturbative approach, this implies that any dependence on $\mu$ in $F_{k}\left(x, Q^{2}\right)$ has to vanish at least to the order in $\alpha_{s}$ considered,

$$
\frac{d}{d \ln \mu^{2}} F_{k}\left(x, Q^{2}\right)=O\left(\alpha_{s}^{l+1}\right) .
$$

Similar arguments, of course, also apply to the dependence on the renormalization scale. General experience shows, that theory uncertainties of better than $O(10 \%)$ according to Eq. (3) for cross sections at hadron colliders (like DIS) require radiative corrections at least to NNLO accuracy in QCD.

Altogether, we are in the comfortable position to confront experimental data with theory at a very high level of precision. In these comparisons, we no longer test QCD. Rather we use perturbative QCD as an essential and established part of our theory toolkit to deduce important information about PDFs or the value of the strong coupling constant $\alpha_{s}\left(M_{Z}\right)$. Of course, this is a situation that, generally, needs to be addressed also beyond DIS, since experimental data from the hadron colliders Tevatron at FNAL and the LHC at CERN help to further constrain the non-perturbative input to QCD precision predictions. For the rest of this review we focus on DIS and kinematically related processes, though. 


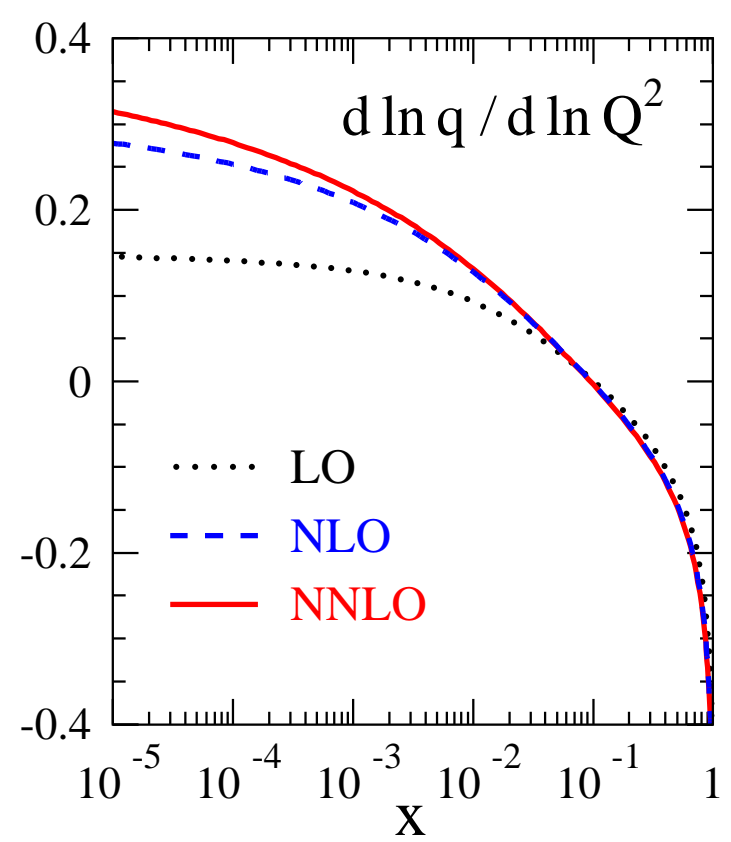

Figure 2: The perturbative expansion of the scale derivatives (6) of the singlet distributions $q \equiv \sum_{i=q, \bar{q}} f_{i}$ and $g \equiv f_{g}$. Plot taken from [26].

\section{Non-perturbative parameters}

The PDFs $f_{i}$ in Eq. (1) are non-perturbative objects as well as the QCD scale $\Lambda_{\mathrm{QCD}}$ or, equivalently the value of the strong coupling constant $\alpha_{s}\left(M_{Z}\right)$ and, also the masses of heavy quarks $m_{q}$, which are not directly observable due to confinement. These fundamental parameters of QCD are indispensable ingredients in any cross section prediction. They have to be obtained from global fits to experimental data or determined, e.g., by lattice computations.

Within perturbation theory, however, using renormalization, some information about their scale dependence is available, once a suitable scheme has been fixed, e.g., the $\overline{\mathrm{MS}}$ scheme, which is nowadays the commonly adopted choice. Then we have for $\alpha_{s}$ the famous QCD beta-function [1] at our disposal,

$$
\frac{d}{d \ln \mu^{2}} \alpha_{s}(\mu)=\beta\left(\alpha_{s}\right),
$$

and the evolution equations for heavy-quark masses

$$
\frac{d}{d \ln \mu^{2}} m_{q}(\mu)=\gamma_{q}\left(\alpha_{s}\right) m_{q}(\mu)
$$

both with perturbatively calculable coefficients known to $\mathrm{N}^{3} \mathrm{LO}$, see [27] for the beta-function coefficients and $[28,29]$ for the mass anomalous dimension $\gamma_{q}$.

Another popular scheme for heavy-quark masses is the so-called on-shell scheme, where the pole-mass $m_{q}$

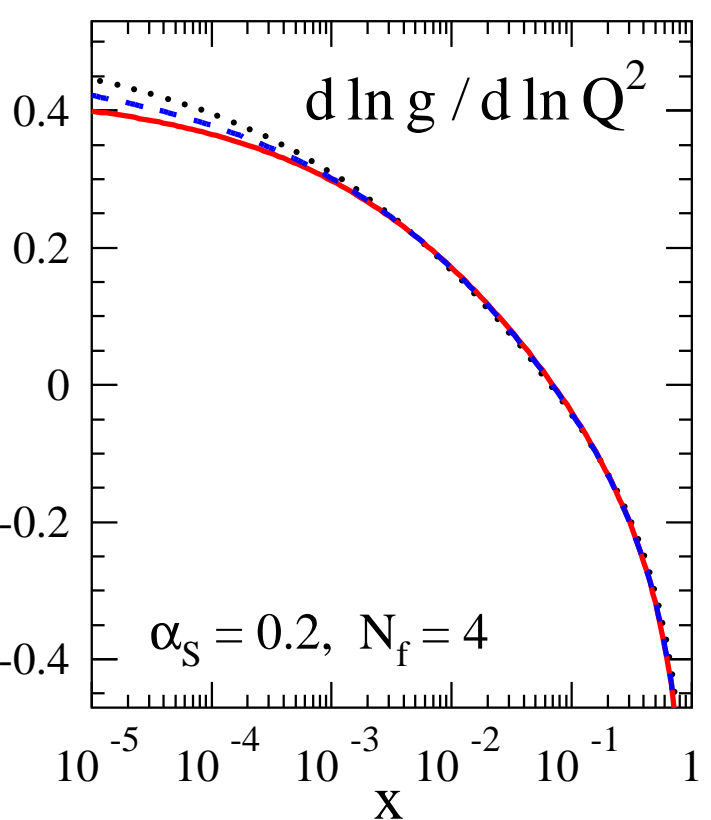

is chosen to coincide with the pole of the heavy-quark propagator at each order in perturbative QCD. Such definition, however, has its intrinsic theoretical limitations with ambiguities of the order $O\left(\Lambda_{\mathrm{QCD}}\right)$ implying a strong dependence of the value of the mass parameter on the order of perturbation theory. The $\overline{\mathrm{MS}}$ scheme Eq. (5) for the heavy-quark mass is favored, because predictions for hard scattering cross sections in terms of the $\overline{\mathrm{MS}}$ mass display better apparent convergence properties and greater perturbative stability at higher orders [30], i.e. features much desired in the computation of radiative corrections as outlined above in Sec. 1.

Finally, for the PDFs we have the well-known evolution equations

$$
\begin{gathered}
\frac{d}{d \ln \mu^{2}}\left(\begin{array}{c}
f_{q_{i}}\left(x, \mu^{2}\right) \\
f_{g}\left(x, \mu^{2}\right)
\end{array}\right)= \\
\sum_{j} \int_{x}^{1} \frac{d z}{z}\left(\begin{array}{cc}
P_{q_{i} q_{j}}(z) & P_{q_{i} g}(z) \\
P_{g q_{j}}(z) & P_{g g}(z)
\end{array}\right)\left(\begin{array}{c}
f_{q_{j}}\left(x / z, \mu^{2}\right) \\
f_{g}\left(x / z, \mu^{2}\right)
\end{array}\right) .
\end{gathered}
$$

where the splitting functions $P_{i j}$ are known to NNLO [31, 26]. These describe the different possible parton splittings in the collinear limit, i.e. the mixing of the respective partonic operators under renormalization, and are, therefore universal quantities. Evolution equations for polarized DIS with spin dependent splitting functions $\Delta P_{i j}$ (known to NLO completely $[32,33]$ and 
for $\Delta P_{q q}$ and $\Delta P_{q g}$ to NNLO [34]) derive from Eq. (6) with the simple replacements $f_{i} \rightarrow \Delta f_{i}$ and $P_{i j} \rightarrow \Delta P_{i j}$.

The perturbative expansion of the evolution Eq. (6) is illustrated in Fig. 2 for the singlet distributions $q \equiv$ $\sum_{i=q, \bar{q}} f_{i}$ and $g \equiv f_{g}$, which displays the apparent convergence upon considering the QCD corrections to the splitting functions through NNLO. Perturbative stability is observed down to values of $x \simeq 10^{-5}$, which is amply enough for the currently accessible range in collider phenomenology. Yet smaller values of $x$ require corrections beyond NNLO or, alternatively, small- $x$ resummations beyond NLL accuracy.

The scale dependence of the fundamental parameters $\alpha_{s}\left(M_{Z}\right)$ and $m_{q}$ as well as for the PDFs $f_{i}$ in Eqs. (4)(6) is used to determine those from global fits to experimental data at a given order in QCD, typically at least to NNLO. Let us briefly comment on the current status in turn. Analyses of the available DIS world data for a determination of $\alpha_{s}\left(M_{Z}\right)$ are performed by a number of groups and summarized in Tab. 1.

\begin{tabular}{|l|l|}
\hline & \multicolumn{1}{|c|}{$\alpha_{s}\left(M_{Z}\right)$} \\
\hline BBG [35] (val.) & $0.1134+0.0019$ \\
ABKM09 [36] & $0.1135 \pm 0.0014$ \\
ABM11 [37] & $0.1134 \pm 0.0011$ \\
JR [38] & $0.1124 \pm 0.0020$ \\
MSTW [39] & $0.1171 \pm 0.0014$ \\
\hline world average (2009) [40] & $0.1184 \pm 0.0007$ \\
(2011) [41] & $0.1183 \pm 0.0010$ \\
\hline
\end{tabular}

Table 1: Summary of recent NNLO QCD analyses of the DIS world data.

The values of $\alpha_{s}\left(M_{Z}\right)$ obtained from the analysis of DIS world data are consistent within their errors with the exception of MSTW [39]. The reason for this difference and also the difference with the world average (cf. Tab. 1) is discussed in great detail in [37]. Measurements of DIS jet cross sections offer an interesting alternative for the extraction of $\alpha_{s}\left(M_{Z}\right)$, although available QCD theory predictions are only accurate to NLO. See $[42,43]$ for the latest analyses.

Available DIS data, especially those from the HERA collider also allows for a determination of the charmquark mass. Starting from inclusive cross section data and a theory description of the heavy quark structure functions $F_{k}\left(x, Q^{2}, m_{c}\right)$ in analogy to Eq. (1) in terms of a running quark mass, cf. Eq. (5), the $\overline{\mathrm{MS}}$ charm-quark mass $m_{c}\left(m_{c}\right)$ has been determined from a variant of the ABKM fit [36]. The phenomenological study of [30] yields to NLO in QCD

$$
m_{c}\left(m_{c}\right)=
$$

$$
1.26 \pm 0.09(\exp ) \pm 0.11 \text { (th) } \mathrm{GeV},
$$

and to approximate NNLO (cf. Sec. 1)

$$
\begin{aligned}
m_{c}\left(m_{c}\right) & = \\
1.01 & \pm 0.09(\exp ) \pm 0.03(\text { th }) \mathrm{GeV},
\end{aligned}
$$

where the quoted experimental uncertainty results from the propagation of the statistical and systematic errors in the data with account of error correlations whenever available while the theoretical uncertainty is estimated from the residual scale dependence, cf. Eq. (3), for the choice $\mu^{2}=Q^{2}+\kappa m_{c}^{2}$ with a range $\kappa \in[0,8]$ These numbers in Eqs. (6) and (7) are to be compared to the world average of the particle data group (PDG) [3] quoted in the $\overline{\mathrm{MS}}$ scheme as $m_{c}\left(m_{c}\right)=1.27_{-0.09}^{+0.07} \mathrm{GeV}$, which is entirely based on lattice computations or analyses of experimental data with time-like kinematics from $e^{+} e^{-}$-collisions, e.g. with the help of QCD sum rules. It is therefore interesting to note that the DIS results in Eqs. (6) and (7) for hadronic processes with spacelike kinematics are consistent with but systematically lower than the PDG value. It should be noted, though, that there is a significant correlation of the value of $m_{c}$ with the value of $\alpha_{s}\left(M_{Z}\right)$ in all analyses currently available which use perturbative QCD results, i.e., QCD sum rules as well as [30].

Last but not least, the available DIS world data allows for a determination of the PDFs using the QCD evolution Eq. (6). To that end, analyses rely on a variety of data predominantly from DIS experiments in order to cover the entire kinematic range in $x$ also combining scattering data with different beams and different targets to allow for the separation of the individual quark flavors.

Of particular interest in applications for a protonproton collider such as the LHC is the gluon PDF as it drives many important search channels, e.g., the cross section for Higgs boson production in gluon-gluon fusion $[44,45]$. It has become obvious, that currently the largest differences between the predictions for the Higgs boson or top-quark pair production of various PDF sets are due to differences in the gluon PDF as well as in the value of $\alpha_{s}\left(M_{Z}\right)$. Fig. 3 illustrates this for the NNLO gluon PDF at a low scale $Q=2 \mathrm{GeV}$ where differences for the gluon in the range $x>0.1$ lead to larger differences at a higher scale of $Q=165 \mathrm{GeV}$ at even smaller $x>0.05$ due to QCD evolution (6), see Fig. 4. These findings are of direct relevance for the production of a Higgs boson in the mass range $M_{H} \simeq O(160) \mathrm{GeV}$. Detailed further discussions of this vast subject and studies on the origin of such differences in PDFs are given in [37]. 


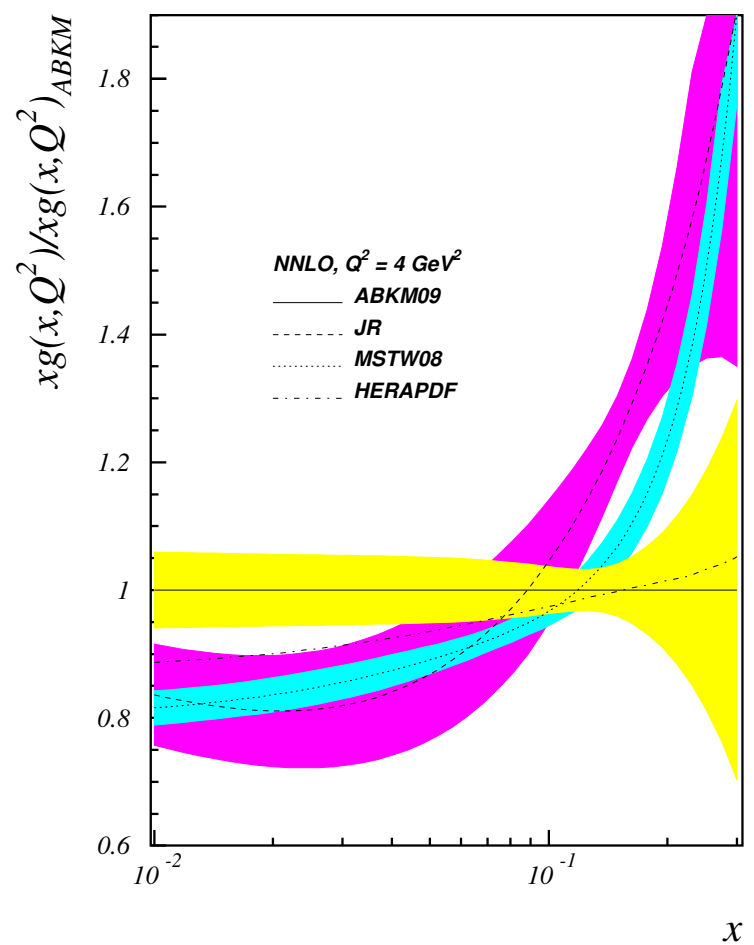

Figure 3: Comparison of the NNLO gluon distributions at $Q^{2}=$ $(2 \mathrm{GeV})^{2}$ for the ratios $x g\left(x, Q^{2}\right) / x g\left(x, Q^{2}\right)_{\mathrm{ABKM}}$ including the $1 \sigma$ uncertainty bands for ABKM [36] (solid line), HERAPDF [46] (dash-dotted line, without error band), JR [38] (dashed line) and MSTW08 [47] (dotted line). Plot taken from [48].

\section{Higgs production via vector-boson fusion}

The production of a Higgs boson via vector-boson fusion (VBF) provides very promising signals for experimental searches with two jets at large rapidity separation and the Higgs decay products being centrally produced in the detector along with little hadronic activity. VBF has the second largest cross section in size. It is a pure electroweak process at LO and it has an interesting relation to DIS.

The QCD radiative corrections to the total crosssection of VBF can be factorized to a very good approximation as a double DIS process, see Fig. 5, where two (virtual) vector-bosons $V_{i}$ (independently) emitted from the hadronic initial states fuse into a Higgs boson. This defines the so-called structure function approach [49], which builds on the absence (or smallness) of the QCD interference between the two inclusive final states $X_{1}$ and $X_{2}$. Then, the total cross section is given as a product of the matrix element for $V_{1} V_{2} \rightarrow H$ and of the DIS hadronic tensor $W_{\mu \nu}$, commonly expressed in terms of the standard DIS structure functions $F_{k}\left(x, Q^{2}\right)$.

Of course, this factorization does not hold exactly

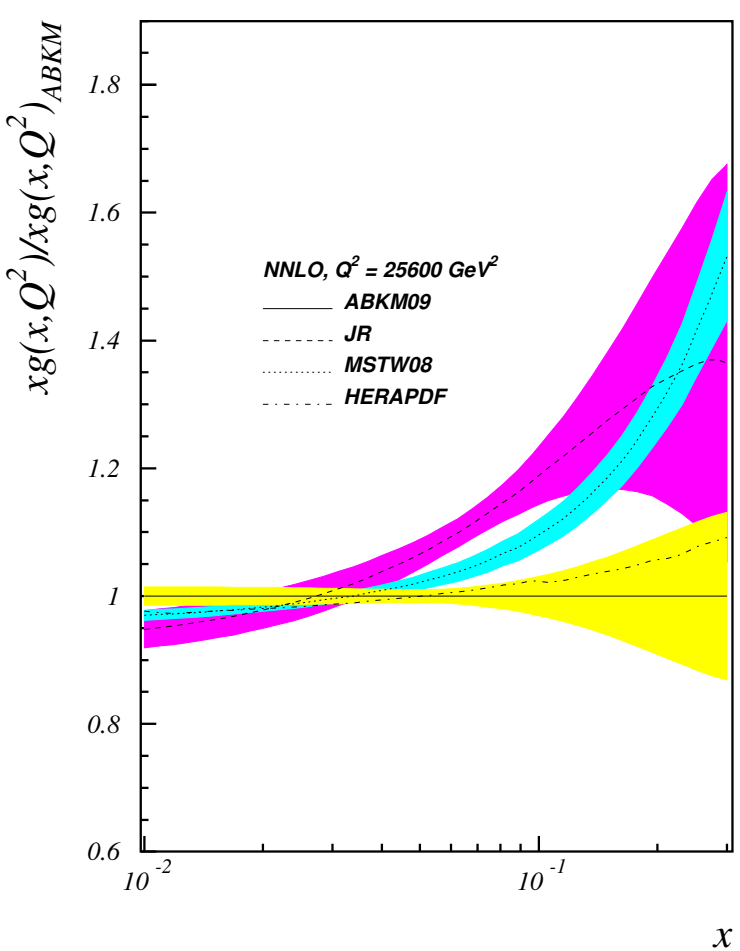

Figure 4: Same as Fig. 4 for at $Q^{2}=(165 \mathrm{GeV})^{2}$. Plot taken from [48].

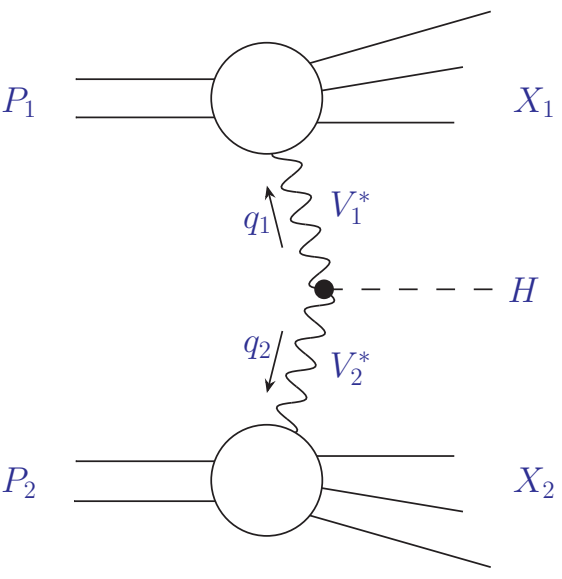

Figure 5: Higgs production via the VBF process.

already at LO, because of interference between identical final state quarks, e.g., ии $\rightarrow$ Нии or between processes where either a $W$ or a $Z$ can be exchanged, e.g., ud $\rightarrow$ Hud. At LO, they can however, be easily computed. The typical accuracy of the NLO QCD predictions can be estimated in the $5-10 \%$ range. The dominant contributions to VBF at NNLO accuracy have been obtained in $[50,51]$. They give rise to dramatic reductions of the theoretical uncertainties, which are sta- 


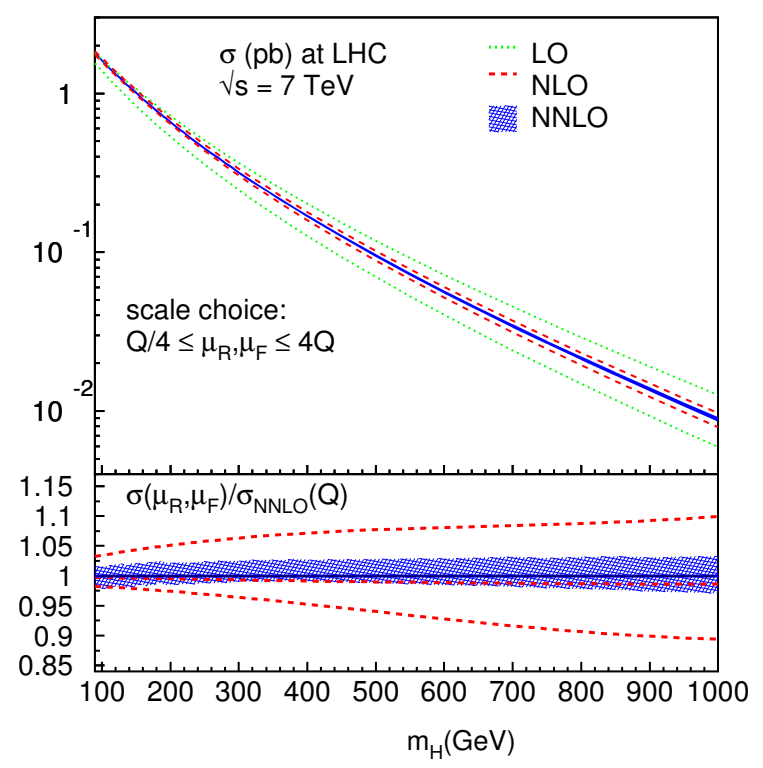

Figure 6: The total VBF cross sections at the LHC, $\sqrt{s}=7 \mathrm{TeV}$, at LO, NLO and NNLO in QCD with the scale uncertainty from the variation $\mu_{r}, \mu_{f} \in[Q / 4,4 Q]$. The MSTW2008 [47] PDF set $(68 \%$ $\mathrm{CL})$ has been used. Numbers are in pb. Plot taken from [50].

bilized at NNLO at the level of $O(2 \%)$, see Fig. 6. This is about the same accuracy as the level of ambiguity at which the Higgs production signal via VBF can be defined phenomenologically due to the particular requirements for the final state.

Although the structure function approach to VBF, is not truly exact at NNLO it includes the bulk of the radiative corrections so that the remaining contributions, are both, parametrically small and kinematically suppressed. Detailed estimates have been provided in [50], so that VBF is currently the most accurately known cross section for Higgs production at the LHC.

Among the uncertainties in the cross section prediction, the PDF dependence is clearly the dominating source of uncertainty, see Fig. 7. We observe that over a large range of Higgs masses, $M_{H} \simeq 100 \ldots 300 \mathrm{GeV}$, there are differences between these sets of order $O(3 \%)$, since the quark PDFs are rather well constrained in the relevant $x$-region. This deteriorates towards larger Higgs masses and, generally, the PDF uncertainties are also larger for the lower running energies of the LHC. Generally, the non-perturbative parameters (PDFs and $\alpha_{s}$ ) needed for precision predictions of Higgs production in VBF are under better control than, e.g., for the gluon-gluon fusion process.

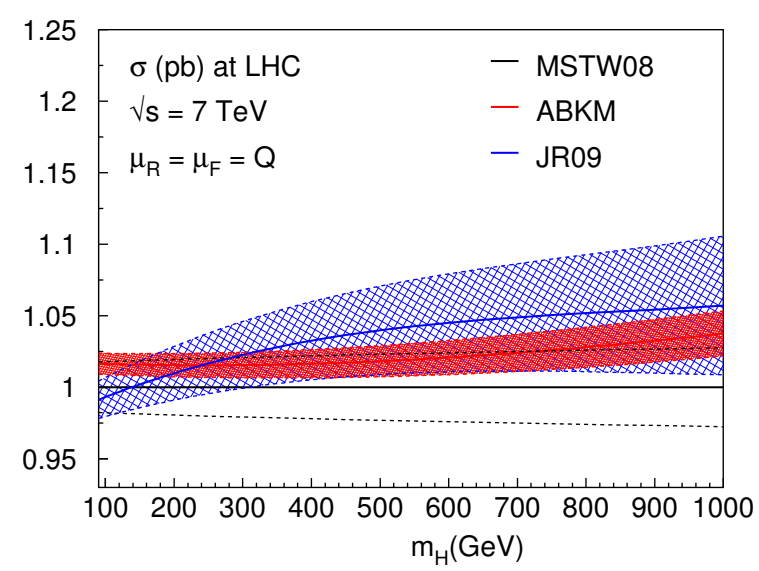

Figure 7: The PDF uncertainty of the VBF cross sections at the LHC, $\sqrt{s}=7 \mathrm{TeV}$, at NNLO in QCD for PDF sets of ABKM [36], JR09 [38, 52] and MSTW2008 [47] (68 \% CL) All results have been normalized to the best fit of MSTW2008. Plot taken from [50].

\section{Summary}

We have briefly summarized the current status of perturbative QCD predictions for observables in DIS. To date, we can build on a very mature understanding of the theory, which is confronted with high precision experimental data in order to provide important information on the dynamics of quarks and gluons in nucleons as well as on the strong coupling constant and heavy quark masses. Especially the data accumulated at HERA has a very high potential in this respect.

We have also discussed, how kinematical situations resembling DIS are found for specific reactions at hadron colliders, e.g., for the Higgs boson production in VBF. In such situations, knowledge on perturbative corrections in DIS can be successfully applied to achieve significantly improved theory predictions.

\section{References}

[1] D. Gross and F. Wilczek, Phys.Rev. D8, 3633 (1973).

[2] D. Gross and F. Wilczek, Phys.Rev. D9, 980 (1974).

[3] Particle Data Group, K. Nakamura et al., J.Phys.G G37, 075021 (2010).

[4] W. van Neerven and E. Zijlstra, Phys.Lett. B272, 127 (1991).

[5] E. B. Zijlstra and W. L. van Neerven, Nucl. Phys. B383, 525 (1992).

[6] E. B. Zijlstra and W. L. van Neerven, Phys. Lett. B297, 377 (1992).

[7] S. Moch and J. Vermaseren, Nucl.Phys. B573, 853 (2000), hep$\mathrm{ph} / 9912355$.

[8] J. Vermaseren, A. Vogt, and S. Moch, Nucl.Phys. B724, 3 (2005), hep-ph/0504242.

[9] S. Moch, J. A. M. Vermaseren, and A. Vogt, Nucl. Phys. B813, 220 (2009), arXiv:0812.4168. 
[10] E. B. Zijlstra and W. L. van Neerven, Nucl. Phys. B417, 61 (1994).

[11] S. Moch, J. Vermaseren, and A. Vogt, Nucl.Phys. B726, 317 (2005), hep-ph/0506288.

[12] T. Becher, M. Neubert, and B. D. Pecjak, JHEP 0701, 076 (2007), hep-ph/0607228.

[13] V. S. Fadin and L. Lipatov, Phys.Lett. B429, 127 (1998), hep$\mathrm{ph} / 9802290$.

[14] E. Laenen et al., Nucl. Phys. B392, 162 (1993).

[15] M. Glück, S. Kretzer, and E. Reya, Phys.Lett. B380, 171 (1996), hep-ph/9603304.

[16] E. Laenen and S. Moch, Phys. Rev. D59, 034027 (1999), hep$\mathrm{ph} / 9809550$.

[17] G. Corcella and A. D. Mitov, Nucl.Phys. B676, 346 (2004), hep-ph/0308105.

[18] N. Presti et al., PoS DIS2010, 163 (2010), arXiv:1008.0951.

[19] A. Daleo, D. de Florian, and R. Sassot, Phys.Rev. D71, 034013 (2005), hep-ph/0411212.

[20] B. Kniehl, G. Kramer, and M. Maniatis, Nucl.Phys. B711, 345 (2005), hep-ph/0411300.

[21] G. Kramer and B. Potter, Eur.Phys.J. C5, 665 (1998), hep$\mathrm{ph} / 9804352$.

[22] Z. Nagy, Phys.Rev.Lett. 88, 122003 (2002), hep-ph/0110315.

[23] Z. Nagy, Phys.Rev. D68, 094002 (2003), hep-ph/0307268.

[24] A. Daleo and R. Sassot, Phys.Rev. D73, 054014 (2006), hep$\mathrm{ph} / 0511189$.

[25] A. Daleo et al., JHEP 1001, 118 (2010), arXiv:0912.0374.

[26] A. Vogt, S. Moch, and J. Vermaseren, Nucl.Phys. B691, 129 (2004), hep-ph/0404111.

[27] T. van Ritbergen, J. Vermaseren, and S. Larin, Phys.Lett. B400, 379 (1997), hep-ph/9701390.

[28] J. Vermaseren, S. Larin, and T. van Ritbergen, Phys.Lett. B405, 327 (1997), hep-ph/9703284.

[29] K. Chetyrkin, Phys.Lett. B404, 161 (1997), hep-ph/9703278.

[30] S. Alekhin and S. Moch, Phys.Lett. B699, 345 (2011), arXiv: 1011.5790 .

[31] S. Moch, J. Vermaseren, and A. Vogt, Nucl.Phys. B688, 101 (2004), hep-ph/0403192.

[32] R. Mertig and W. van Neerven, Z.Phys. C70, 637 (1996), hep$\mathrm{ph} / 9506451$.

[33] W. Vogelsang, Nucl.Phys. B475, 47 (1996), hep-ph/9603366.

[34] A. Vogt et al., Nucl.Phys.Proc.Suppl. 183, 155 (2008), arXiv:0807.1238.

[35] J. Blümlein, H. Böttcher, and A. Guffanti, Nucl.Phys. B774, 182 (2007), hep-ph/0607200.

[36] S. Alekhin et al., Phys.Rev. D81, 014032 (2010), arXiv:0908.2766.

[37] S. Alekhin, J. Blümlein, and S. Moch, (2012), in preparation.

[38] P. Jimenez-Delgado and E. Reya, Phys.Rev. D79, 074023 (2009), arXiv:0810.4274

[39] A. D. Martin et al., Eur. Phys. J. C64, 653 (2009), arXiv:0905.3531

[40] S. Bethke, Eur. Phys. J. C64, 689 (2009), arXiv:0908.1135.

[41] S. Bethke et al., (2011), arXiv:1110.0016.

[42] G. Grindhammer, (2011), arXiv:1112.0224.

[43] R. Kogler, (2011), arXiv:1112.5117.

[44] J. Baglio and A. Djouadi, JHEP 1010, 064 (2010), arXiv:1003.4266.

[45] S. Alekhin, J. Blümlein, and S. Moch, Eur.Phys.J. C71, 1723 (2011), arXiv:1101.5261.

[46] H1 and ZEUS Collaboration, F. Aaron et al., JHEP 1001, 109 (2010), arXiv:0911.0884.

[47] A. Martin et al., Eur.Phys.J. C63, 189 (2009), arXiv:0901.0002.

[48] S. Alekhin et al., Phys.Lett. B697, 127 (2011), arXiv:1011.6259.
[49] T. Han, G. Valencia, and S. Willenbrock, Phys.Rev.Lett. 69, 3274 (1992), hep-ph/9206246.

[50] P. Bolzoni et al., (2011), arXiv:1109.3717.

[51] P. Bolzoni et al., Phys.Rev.Lett. 105, 011801 (2010), arXiv: 1003.4451

[52] P. Jimenez-Delgado and E. Reya, Phys.Rev. D80, 114011 (2009), arXiv:0909.1711. 